\title{
Twelve novel FBN1 mutations in Marfan syndrome and Marfan related phenotypes test the feasibility of FBN1 mutation testing in clinical practice
}

\author{
D J Halliday, S Hutchinson, L Lonie, J A Hurst, H Firth, P A Handford, P Wordsworth
}

J Med Genet 2002;39:589-593

M arfan syndrome (MFS) is one of the major heritable disorders of connective tissue with a prevalence of between 1 in 5-10 000. ${ }^{12}$ It is characterised by features in the cardiovascular, ocular, and musculoskeletal systems and the Ghent criteria form a useful framework for its diagnosis. ${ }^{3}$ Mutations in FBNI encoding the extracellular matrix protein fibrillin-1 classically cause MFS. ${ }^{4}$ Fibrillin- 1 , comprising multiple repeating subunits, of which the most common is the calcium binding epidermal growth factor (cbEGF) domain, is a key component of 10-12 nm microfibrils. ${ }^{5}$

Mutation detection has not been performed routinely in MFS because of the size and complexity of FBN1, which contains 65 exons extending over $200 \mathrm{~kb}$ of genomic DNA. ${ }^{5}$ Mutations are nearly always specific to each family and to date 300 mutations have been reported. ${ }^{7-10}$ FBNI mutations have been found in $20-80 \%$ of patients with MFS depending upon the clinical selection of patients and the mutation detection method used. ${ }^{11-17}$ FBNI mutations have been reported in a wide range of phenotypes in the Marfan spectrum'; neonatal MFS is associated with some FBNI mutations in exons 24-32 ${ }^{18}$; mutations of various types throughout FBN1 have been associated with classical MFS ${ }^{8}$; and FBN1 mutations have also been reported in many of the non-classical Marfan related phenotypes. ${ }^{1}$ The Ghent criteria propose that an FBNI mutation is only of help diagnostically if it has been previously found in a person who independently meets the criteria for diagnosis of MFS. ${ }^{3}$

Other than mutations causing neonatal MFS, which occur in exons 24-32, correlation between genotype and phenotype is poor. The majority of patients with cysteine substitutions have classical MFS, and cysteine substitutions in exons 26-32 appear to be associated with classical disease manifesting early in life. ${ }^{19}$ Missense mutations that affect the calcium binding site of the cbEGF domain (reducing calcium binding affinity) are associated with varying phenotypes that are likely to be related to the location, calcium binding properties, and domain context of the affected domain within fibrillin-l. There is marked phenotypic variation between subjects with nonsense mutations, which is thought to be due to the degree of nonsense mediated decay of the mutant cDNA. ${ }^{20}$ Intrafamilial variation of phenotype is also seen, suggesting that there are factors in addition to the FBN1 genotype that can modify the phenotype. ${ }^{21} 22$ Consequently, knowledge of the underlying FBN1 mutation in an affected proband is of relatively little help either in assigning the appropriate clinical diagnosis or in advising on the likely clinical course or severity of phenotype. Here we report our experience in the Oxford Marfan Clinic, where mutation detection has been performed on 35 families on a research basis using heteroduplex analysis of genomic amplicons with conformation sensitive gel electrophoresis (CSGE) or denaturing high performance liquid chromatography (DHPLC).

\section{METHODS}

The clinical features of the probands are given in table 1 . All patients underwent a thorough clinical examination, including slit lamp examination and 2D echocardiogram. Magnetic resonance imaging (MRI) of the lumbosacral spine for evidence of dural ectasia was performed in 14 of the probands. Of the 35 probands, 22 fulfilled the Ghent criteria for MFS (table 1). Two further subjects had two major criteria but involvement of a third system was not demonstrated. The remaining 11 subjects had phenotypes related to MFS. Other family members were also examined for Marfan syndrome where practicable.

Mutation detection was performed initially on 17 subjects using heteroduplex analysis of genomic amplicons and CSGE with DNA sequencing, as has been previously described. ${ }^{23}$ In subject 12 , where a mutation had not been discovered using CSGE, the cDNA was directly sequenced, as a defect had been observed in fibrillin-1 pulse chase studies. ${ }^{24}$ DHPLC was used to screen for mutations in the remainder of the patients, including five cases where a mutation had not been found previously using CSGE (cases 2, 5, 7, 15, and 19). Sensitivity of the conditions used for DHPLC were tested by ensuring that DHPLC was able to identify 13 known FBN1 mutations or polymorphisms which had been previously detected by CSGE. The 14 mutation negative subjects only analysed with DHPLC were not rescreened using CSGE, as DHPLC was the more

\section{Key points}

- Marfan syndrome is caused by mutations in FBNI resulting in defective fibrillin-1. It is diagnosed mainly on clinical grounds although FBN1 genetic analysis may contribute.

- We have performed mutation detection on 35 families from the Oxford Marfan Clinic, with either classical Marfan syndrome or a Marfan related phenotype; 21 mutations were identified, of which 10 are reported here for the first time.

- In this study we found an FBN1 mutation detection rate of $70 \%$ when this analysis was restricted to those with at least one major clinical criterion lexcluding family history) and involvement of one further system.

- We suggest that this would be a suitable clinical requirement before $F B N 1$ testing in clinical practice.

Abbreviations: MFS, Marfan syndrome; cbEGF, calcium binding epidermal growth factor; CSGE, conformation sensitive gel electrophoresis; DHPLC, denaturing high performance liquid chromatography 
Table 1 Details of the clinical features of the probands used in mutation detection along with details of the exon location, the nucleic acid change, the predicted consequence, the domain of fibrillin- 1 , and the method used in detection of each mutation

\begin{tabular}{|c|c|c|c|c|c|c|c|c|c|c|c|c|c|}
\hline \multirow[b]{2}{*}{ Patient } & \multirow[b]{2}{*}{ Age } & \multicolumn{6}{|c|}{ Clinical features* } & \multirow[b]{2}{*}{ Diagnosis† } & \multirow[b]{2}{*}{ Exon } & \multirow[b]{2}{*}{ Mutation $\ddagger$} & \multirow{2}{*}{$\begin{array}{l}\text { Predicted } \\
\text { consequence }\end{array}$} & \multirow{2}{*}{$\begin{array}{l}\text { Fibrillin-1 } \\
\text { domain }\end{array}$} & \multirow[b]{2}{*}{$\begin{array}{l}\text { Method of } \\
\text { analysis }\end{array}$} \\
\hline & & $M / S$ & CVS & Ocular & $\mathrm{DE}$ & $\mathrm{FH}$ & minor & & & & & & \\
\hline 11 & 46 & - & $+(A D)$ & - & + & $\mathrm{F}+$ & 2 & MFS & 2 & $G 247+1 A \S^{* *}$ & Skip exon 2 & $\mathrm{NH}_{2}$ & CSGE \\
\hline 3 & 45 & - & + & - & + & $\mathrm{F}+$ & 2 & MFS & 8 & 932 insT§ * * & PTC at 348 & cbEGF2 & CSGE \\
\hline 7 & 65 & - & - & + & D & $\mathrm{F}$ - & 1 & $\mathrm{FEL}$ & 15 & C1879T** & R627C†t & cbEGF6 & DHPLC \\
\hline 33 & 40 & - & + & - & + & $\mathrm{F}$ - & 2 & MFS & 15 & G1960A & D654N & TB2 & DHPLC \\
\hline 36 & 54 & - & $+(A D)$ & + & & S & 2 & MFS & 18 & G2168-1A & Splice defect & cbEGF7 & DHPLC \\
\hline 40 & 9 & - & + & + & $D$ & S & 0 & MFS- & 18 & G2243A & C748Y & cbEGF7 & CSGE \\
\hline 21 & 38 & + & - & + & + & $\mathrm{F}+$ & 0 & MFS & 20 & G2539+1A & Splice defect & cbEGF9 & DHPLC \\
\hline 18 & 30 & - & + & - & + & $\mathrm{F}$ & 2 & MFS & 35 & $4365 \mathrm{delCT} \S$ & PTC at 1470 & cbEGF21 & CSGE \\
\hline 17 & 31 & + & + & - & & $\mathrm{F}$ & 1 & MFS & 36 & 4485 delC $\S$ & PTC at 1519 & cbEGF22 & CSGE \\
\hline 13 & 43 & - & + & + & & $\mathrm{F}$ & 3 & MFS & 37 & C4621T§** & R1541X & TB4 & CSGE \\
\hline 14 & 31 & - & + & + & & $S$ & 2 & MFS & 44 & G5504A§ & C1835Y & cbEGF26 & CSGE \\
\hline 15 & 30 & - & + & + & D & $\mathrm{S}$ & 0 & MFS- & 45 & A5546-2G & Splice defect & cbEGF27 & DHPLC \\
\hline 12 & 25 & + & + & + & & $\mathrm{F}$ & 0 & MFS & 47 & G5788+1A & Ins 33 bases & cbEGF29 & Sequencing \\
\hline 41 & 10 & - & + & + & & $S$ & 1 & MFS & 48 & T5929C & C1977R & cbEGF30 & CSGE \\
\hline 1 & 46 & - & + & + & + & $\mathrm{F}+$ & 1 & MFS & 55 & G6773A $\S^{* *}$ & C2258Y & cbEGF 35 & CSGE \\
\hline 2 & 43 & + & + & + & & $\mathrm{F}-$ & 0 & MFS & 57 & A6998-2G* * & Del 12 bases & TB7 & DHPLC \\
\hline 16 & 43 & - & $+(A D)$ & + & & $S$ & 3 & MFS & 57 & C7180T§ & R2394X & TB7 & CSGE \\
\hline 26 & 37 & + & - & - & + & $\mathrm{S}$ & 1 & MFS & 59 & C7398A & 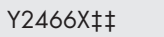 & cbEGF38 & DHPLC \\
\hline 24 & 52 & - & + & + & & $\mathrm{F}$ & 2 & MFS & 59 & C7399T & Q2467X & cbEGF38 & DHPLC \\
\hline 42 & 40 & - & + & - & + & $S$ & 2 & MFS & 62 & 7816 insT & PTC at 2607 & cbEGF42 & CSGE \\
\hline 27 & 17 & - & - & + & D & $\mathrm{F}$ - & 2 & $\mathrm{FEL}$ & 65 & T8339C** & L2780P & C-term & DHPLC \\
\hline 29 & 55 & + & - & - & + & $S$ & 2 & MFS & & - & & & DHPLC \\
\hline 30 & 25 & + & + & - & + & $\mathrm{F}$ & 0 & MFS & & - & & & DHPLC \\
\hline 5 & 62 & - & + & + & & $\mathrm{F}+$ & 1 & MFS & & - & & & DHPLC \\
\hline 6 & 41 & + & $+(A D)$ & - & + & $\mathrm{F}-$ & 0 & MFS & & - & & & DHPLC \\
\hline 19 & 48 & + & - & - & + & $S$ & 1 & MFS & & - & & & DHPLC \\
\hline 34 & 50 & - & - & + & & $\mathrm{S}$ & 0 & $\mathrm{EL}$ & & - & & & DHPLC \\
\hline 22 & 38 & - & + & - & $D$ & $\mathrm{~F}$ & 2 & FTAA & & - & & & DHPLC \\
\hline 25 & 41 & - & + & - & D & $\mathrm{F}$ & 1 & FTAA & & - & & & DHPLC \\
\hline 23 & 48 & + & - & - & D & $\mathrm{F}$ - & 1 & FMH & & - & & & DHPLC \\
\hline 38 & 46 & - & - & - & + & $\mathrm{F}$ & 3 & MASS & & - & & & DHPLC \\
\hline 31 & 53 & - & - & - & - & $\mathrm{F}$ - & 3 & MASS & & - & & & DHPLC \\
\hline 37 & 32 & - & - & - & & $S$ & 1 & Striae & & - & & & DHPLC \\
\hline 35 & 27 & - & - & - & & $\mathrm{F}$ - & 1 & - & & - & & & DHPLC \\
\hline 32 & 10 & - & $+(A D)$ & - & & $S$ & 0 & SG & & - & & & DHPLC \\
\hline
\end{tabular}

*Clinical features. Musculoskeletal (M/S), cardiovascular (CVS), ocular and dural ectasia (DE) are marked with a + if features were found on examination that corresponded to major involvement by the Ghent criteria. ${ }^{3}$ The aortic root was considered dilated when the maximum diameter at the sinus of Valsalva exceeded published normograms for age and body surface area $\left(95 \%\right.$ normal confidence limits). ${ }^{39} \mathrm{AD}$ indicates a previous aortic dissection. Dural ectasia was diagnosed based on published recommendations. ${ }^{40}+=\mathrm{DE}$ present, - = DE absent, blank MRI scan not performed, D MRI declined (7 and 27 ), delayed (22 and 25) because of aortic root surgery or prevented because of spinal deformity (23), age (40), or lost to follow up (15), but all seven of these subjects would fulfil the Ghent criteria for MFS if dural ectasia were present. FH, family history. F, familial phenotype. S, sporadic phenotype. + indicates that at least one relative was examined and fulfilled the Ghent criteria independently. - indicates that the relatives were examined and did not fulfil the Ghent criteria independently. The number of systems where minor criteria (such as $\mathrm{M} / \mathrm{S}$ features, mitral valve prolapse, striae, or pneumothorax) were found (excluding those systems which also had a major criterion) is indicated. Keratometry and ultrasound of the globe to look for minor opthalmological involvement was not performed routinely.

†Diagnosis. The diagnosis of MFS was made according to the Ghent criteria if there were at least two major criteria and minor involvement of a third system; -, third system involvement was not demonstrated in these subjects. FEL, familial ectopia lentis; EL ectopia lentis - sporadic; FTAA, familial thoracic aortic aneurysm; FMH, familial Marfan-like habitus; SG, Shprintzen-Goldberg syndrome based on features of fatal aortic dissection, craniosynostosis, join laxity, and arthrogryposis multiplex congenita. Two subjects had just one minor Ghent criterion but were included because of the familial nature of mild Marfan features in case 35, where four subjects had minor musculoskeletal features of MFS, and the severity of the sporadic Marfan-like striae in subject 37.

$\ddagger$ Nucleic acid and amino acid numbering according to Pereira et al. ${ }^{5}$ PTC, premature termination codon; Ins, insertion; Del, deletion.

$\S$ Published previously. ${ }^{23}$

IPublished previously. ${ }^{24}$

* FBN1 mutation also found in affected relatives.

††Reported previously.

$\ddagger \ddagger$ Reported previously.

sensitive technique in our laboratory, as shown by its ability to detect three mutations (cases 2, 7, and 15) that had not been detected using CSGE.

\section{DHPLC analysis}

All 65 exons of FBN1 and their flanking intronic regions were amplified by PCR from genomic DNA. Primers used were as published $^{13}$ except for 42,47 , and 49 , where the primers were redesigned (table 2 ). PCR was performed in a $50 \mu \mathrm{l}$ volume containing $30 \mathrm{ng}$ of genomic DNA, $1 \times$ PCR buffer II ( 100 $\mathrm{mmol} / \mathrm{l}$ Tris- $\mathrm{HCl}, \mathrm{pH} 8.3,500 \mathrm{mmol} / \mathrm{l} \mathrm{KCl}$ ) (Perkin Elmer), 0.2 $\mathrm{mmol} / \mathrm{l} \mathrm{dNTPs}, 1.5-3.0 \mathrm{mmol} / \mathrm{l} \mathrm{MgCl}_{2}$, $50 \mathrm{ng}$ each primer, and 0.05 U Pfu Turbo DNA polymerase (Stratagene) mixed with 0.9 U AmpliTaq gold DNA polymerase (PE-Applied Biosystems), and was amplified using a touchdown PCR cycle on a PTC 225 Thermal Cycler (MJ Research Inc, Massachusetts, USA). Mg2 ${ }^{+}$ concentration and annealing temperature were optimised for each primer pair and were usually between 1.5-3.0 mmol/l $\mathrm{MgCl}_{2}$ with an annealing temperature of $50-58^{\circ} \mathrm{C}$. To encourage formation of heteroduplexes, the PCR products were denatured at $95^{\circ} \mathrm{C}$ for five minutes and cooled gradually to $25^{\circ} \mathrm{C}$ at $-1^{\circ} \mathrm{C}$ per minute.

DHPLC analysis was carried out using the WAVE ${ }^{\mathrm{TM}}$ DNA Fragment Analysis System (Transgenomic, Cheshire, UK). The temperatures for DHPLC analysis were determined using the Transgenomic WAVEMaker ${ }^{\mathrm{TM}} 3.4$ program (table 3 ) and are available on request. The PCR products were injected into a DNASep column (Transgenomic). The column mobile phase 
Table 2 Details of the primer sequences that were redesigned to allow improved amplification of $F B N 1$ exons 42, 47, and 49

\begin{tabular}{l} 
Primer sequence \\
\hline 42 f 5'-TATCCTCCGGTCCCACCT \\
42 r 5'-AAAGTTCTGACAATGCCGTC \\
47 f 5'-GGAATTGTTGGGGACTTTTCT \\
47 r 5'-GGTCTTTCTAAGTCCTGTAC \\
49 f 5'-TGATGTCTCCATCGTGTTTGAC \\
49 r 5'-AGACCACCACAAATAAACATGCA
\end{tabular}

consisted of a linear acetonitrile gradient in a $0.1 \mathrm{~mol} / \mathrm{l}$ triethylamine acetate buffer (TEAA), achieved by mixing buffers A ( $0.1 \mathrm{~mol} / \mathrm{l} \mathrm{TEAA})$ and B (25\% acetonitrile in $0.1 \mathrm{~mol} / \mathrm{l}$ TEAA). The calculated gradient (at a flow rate of 0.9 $\mathrm{ml} /$ minute over the six minute cycle) was run for all the amplicons, at the relevant column temperatures for each fragment. Where the chromatograph peak was different from the control, indicating a potential heteroduplex, the fragment was PCR amplified using the same primers and sequenced as has been previously described. ${ }^{23}$

\section{RESULTS}

Table 1 gives the results of FBNI mutation analysis. A total of 21 mutations were detected in the 35 families. Seventeen of the mutations were detected in patients who strictly fulfilled the Ghent criteria for MFS, two mutations were found in subjects with familial ectopia lentis, and two mutations were detected in subjects with only two demonstrable major criteria for MFS. Mutations were not detected in five further subjects with classical MFS (two of whom were screened using both CSGE and DHPLC) or the remaining nine subjects with phenotypes related to MFS. The FBNI mutations comprised seven missense mutations, four nonsense mutations, four frameshift mutations, and six splice site mutations (predicted to lead to exon skipping in four cases and shown in one case to cause a large insertion of 33 bases in the RNA transcript, and in one case to cause a deletion of the first 12 bases of exon 57). Three silent mutations (C510T, T8502C, and A3963G) were detected that did not appear to have functional significance along with eight previously identified polymorphisms: C306T, T1875C, A2168-46G, 3589+15del5bp, C506635T, A6496+128G, G6997+17C, and T7571-113C. $.^{13} 1625-27$

\section{DISCUSSION}

Twenty-one predicted causal mutations were detected in patients from the Oxford Marfan Clinic. Causation is suggested by the type of mutation found (cysteine, nonsense, frameshift, and splice site), since these types of mutation are known to alter the structure and/or amount of fibrillin-1. Further evidence of cause and effect is suggested in cases 1, 2, 3, and 11 , where in these large families the mutation was found to segregate with the disease phenotype (data not shown). The detected mutations occurred throughout the length of FBNl and are typical of those found in other MFS patients. ${ }^{8}$ $F B N 1$ mutations have been reported only rarely in ectopia lentis. ${ }^{102829}$ In this study FBN1 mutations were detected in two probands with familial ectopia lentis (cases 7 and 27). Case 7 (with mutation R627C) and her daughter both had ectopia lentis. For both subjects the aortic root diameter at the sinus of Valsalva was within the normal range for age and body surface area. Mitral valve prolapse was found in the daughter only. Musculoskeletal involvement included positive wrist and thumb signs in both subjects and, in addition, the mother also had a disproportionate body habitus with an
Table 3 Details of the column temperatures used in DHPLC analysis. FBN1 mutations that had been previously identified using CSGE were used as controls to ensure that the temperatures selected for analysis were appropriate. Wherever a heteroduplex resulting from either a known mutation, new mutation, or polymorphism was identified, the temperature at which the heteroduplex was detected is indicated in bold

\begin{tabular}{|c|c|c|c|}
\hline Exon & $\begin{array}{l}\text { Column } \\
\text { temperature }\left({ }^{\circ} \mathrm{C}\right)\end{array}$ & Exon & $\begin{array}{l}\text { Column } \\
\text { temperature }\left({ }^{\circ} \mathrm{C}\right)\end{array}$ \\
\hline 1 & 6264 & 34 & 62 \\
\hline 2 & 5658 & 35 & 62 \\
\hline 3 & 5661 & 36 & 60 \\
\hline 4 & 5759 & 37 & 5961 \\
\hline 5 & 5861 & 38 & 5659 \\
\hline 6 & 596265 & 39 & 555961 \\
\hline 7 & 5860 & 40 & 5760 \\
\hline 8 & 5560 & 41 & 565861 \\
\hline 9 & 606264 & 42 & 60 \\
\hline 10 & 556063 & 43 & 5860 \\
\hline 11 & 6062 & 44 & 6064 \\
\hline 12 & 576063 & 45 & 5456 \\
\hline 13 & 5759 & 46 & 5759 \\
\hline 14 & 58 & 47 & 5961 \\
\hline 15 & 6062 & 48 & 59 \\
\hline 16 & 61 & 49 & 5761 \\
\hline 17 & 5962 & 50 & 596164 \\
\hline 18 & 5457 & 51 & 61 \\
\hline 19 & 57 & 52 & 5458 \\
\hline 20 & 5558 & 53 & 59 \\
\hline 21 & 5461 & 54 & 59 \\
\hline 22 & 56 & 55 & 5861 \\
\hline 23 & 5459 & 56 & 5663 \\
\hline 24 & 576163 & 57 & 5562 \\
\hline 25 & 5861 & 58 & 5458 \\
\hline 26 & 5962 & 59 & 59 \\
\hline 27 & 5863 & 60 & 60 \\
\hline 28 & 5862 & 61 & 5962 \\
\hline 29 & 565961 & 62 & 6064 \\
\hline 30 & 58 & 63 & 6062 \\
\hline 31 & 5559 & 64 & 61 \\
\hline 32 & 59 & 65 & 5658 \\
\hline 33 & 5660 & & \\
\hline
\end{tabular}

upper segment to lower segment ratio of 0.85 , and a high palate with crowding of the teeth. Lumbosacral MRI excluded the presence of dural ectasia in the daughter. Case 27 nearly meets diagnostic requirements for MFS with ectopia lentis, musculoskeletal features (a disproportionate body ratio, positive wrist and thumb signs, pes planus, joint hypermobility, and a highly arched palate with dental crowding), and striae. Protrusio acetabulae was not present, neither was there cardiovascular involvement. The patient declined lumbosacral MRI. The mother of this subject had bilateral ectopia lentis, a non-dilated aortic root, and a mild pectus excavatum. They both carried the L2780P mutation. The sister of the proband also carried L2780P but had only pes planus, a highly arched palate with crowding of the teeth, and striae. Her lenses were not dislocated. More fibrillin-1 functional studies are needed to determine how FBN1 mutations such as these cause a less severe phenotype.

The detection rate of FBNI mutations in this study group overall was $21 / 35(60 \%)$. In those who fulfilled the Ghent criteria for MFS, the detection rate was $17 / 22$ (77\%). This is broadly compatible with previous reports in patients fulfilling the Ghent criteria for MFS of 56\% (34/61) using heteroduplex analysis with DHPLC, ${ }^{16} 72 \%(48 / 66),{ }^{30}$ and $90 \%(18 / 20)^{10}$ using heteroduplex analysis with CSGE, $80 \%(8 / 10)^{17}$ using single strand conformation analysis, and 66\% (62/94) using CSGE or single strand conformation polymorphism (SSCP). ${ }^{9}$ Based on these six studies, heteroduplex analysis of genomic amplicons 
using a technique such as DHPLC might be expected to give an FBN1 mutation detection rate of up to $68 \%$ (187/273) in subjects fulfilling the Ghent criteria. In our population, the detection rate for those who had a Marfan related phenotype was only $2 / 11(18 \%)$, despite the fact that they were all analysed using DHPLC, which has been shown to be a highly sensitive technique for mutation detection in FBNI and other genes. ${ }^{27}{ }^{31}{ }^{32}$ This is consistent with the previously reported figures of 5/32 (15\%) in Marfan related phenotypes, analysed using DHPLC ${ }^{16}$ or 9/77 (12\%) using CSGE or SSCP. It should be noted that analysis of genomic amplicons will not detect mutations caused by changes in the regulatory or promoter regions of $F B N 1$ or deletion of a whole gene. Further analysis of the fibrillin-1 protein or of the fibrillin-1 cDNA may show mutations in those patients where an FBNl mutation has not been detected in the coding sequence. ${ }^{14} 242733$

Collectively this and previous studies confirm that the majority of those with a detectable FBNI mutation have clear clinical features of MFS. Mutation detection can therefore often confirm an underlying defect in fibrillin- 1 in those with a clinical diagnosis of classical MFS, but uncommonly in those with a Marfan related phenotype. It is clear from the observation that clinical features, such as aortic dissection, often occur in several subjects within a family, ${ }^{34}$ that individual mutations do correlate with the phenotype. However, knowledge of the mutation alone is usually insufficient to predict the phenotype in isolation from clinical observations in other family members. With the lack of correlation between genotype and phenotype, ${ }^{95}$ precise knowledge of the particular underlying FBN1 mutation in a proband does not help with prognosis or affect management decisions.

The principal clinical benefit of detecting an FBNI mutation in a patient is the direct molecular test that it offers to relatives. Where an FBNI mutation is detected, the first line investigation for relatives can become mutation detection rather than echocardiography and slit lamp examination. This can be particularly helpful in the screening of children at an early stage and potentially may save them and their families several years of repeated screening and anxiety. It may also help in the investigation of families dispersed geographically and managed by different centres. Knowledge of the FBN1 mutation also allows the possibility of prenatal or preimplantation genetic diagnosis. ${ }^{36}$ In large families, linked markers can be used to show that the phenotype segregates with a particular haplotype, and this approach has been advocated for service use. ${ }^{37}{ }^{38}$ However, this method typically requires large numbers of family samples and is of no use in the $\sim 30 \%$ of cases that are sporadic.

Our data, taken with the results of previous studies, indicate that for routine service there would be a high detection rate of FBN1 mutations using heteroduplex analysis of genomic amplicons by DHPLC, provided that those selected for analysis had clear features of Marfan syndrome. We suggest that a minimum requirement of one major Ghent criterion (excluding family history) and involvement of a second system in a patient would be appropriate. This would allow the detection of the majority of FBN1 mutations identifiable by this method, yet still be compatible with a reasonable detection rate. In this study it would have given a detection rate of $21 / 30(70 \%)$. Where a mutation was not detected by heteroduplex analysis of genomic amplicons by DHPLC, further analysis at the cDNA level could be performed if there was a particular need to know the causative mutation, such as for use in prenatal diagnosis. This could involve restriction enzyme analysis to identify the presence of a large insertion or deletion in FBNI, quantitation of the proportion of transcript from each allele (where an unequal ratio of transcript could indicate an underlying FBN1 mutation), or a direct search for the mutation by sequencing the FBNI cDNA. ${ }^{14} 2733$

In summary, knowledge of the particular FBNI mutation offers little direct benefit for prognosis and management to the person tested, other than in prenatal diagnosis. However, FBN1 mutation testing allows confirmation of the underlying genetic defect and allows its use for diagnosis in relatives, enabling a precise diagnosis in relatives with equivocal features. Precise early molecular diagnosis could obviate the need for repeated clinical screening including echocardiography and slit lamp examination, thereby offsetting the cost of mutation analysis. FBNI testing at the service level could therefore be a cost effective investigation.

\section{ACKNOWLEDGEMENTS}

DH was funded by a British Heart Foundation Junior Research Fellowship and $\mathrm{SH}$ by a BBSRC studentship. PH acknowledges support from the British Heart Foundation and the Medical Research Council. We thank Ann Roberts for assistance with the Marfan clinic.

\section{Authors' affiliations}

D J Halliday, J A Hurst, Department of Clinical Genetics, The Churchill Hospital, Old Road, Headington, Oxford OX3 7L, UK

S Hutchinson, P A Handford, Division of Molecular and Cellular Biochemistry, Department of Biochemistry, University of Oxford, South Parks Road, Oxford OX1 3QU, UK

L Lonie, P Wordsworth, Wellcome Trust Centre for Human Genetics, University of Oxford, Roosevelt Drive, Oxford, UK

H Firth, Department of Medical Genetics, Cambridge University, Cambridge CB2 2QQ, UK

Correspondence to: Dr D J Halliday, Department of Clinical Genetics, The Churchill Hospital, Old Road, Headington, Oxford OX3 7L, UK: penny@bioch.ox.ac.uk

\section{REFERENCES}

1 Dietz HC, Pyeritz RE. Mutations in the human gene for fibrillin-1 (FBN1) in the Marfan syndrome and related disorders. Hum Mol Genet 1995; 4:1799-809

2 Gray JR, Davies SJ. Marfan syndrome. J Med Genet 1996;33:403-8.

3 De Paepe A, Devereux RB, Dietz HC, Hennekam RC, Pyeritz RE. Revised diagnostic criteria for the Marfan syndrome. Am J Med Genet 1996:62:417-26

4 Dietz HC, Cutting GR, Pyeritz RE, Maslen CL, Sakai LY, Corson GM, Puffenberger EG, Hamosh A, Nanthakumar EJ, Curristin SM, Stetten G Meyers DA, Francomano CA. Marfan syndrome caused by a recurrent de novo missense mutation in the fibrillin gene. Nature 1991:352:337-9.

5 Pereira L, D'Alessio M, Ramirez F, Lynch JR, Sykes B, Pangilinan T, Bonadio J. Genomic organization of the sequence coding for fibrillin, the defective gene product in Marfan syndrome. Hum Mol Genet 1993;2:961-8.

6 Biery NJ, Eldadah ZA, Moore CS, Stetten G, Spencer F, Dietz HC. Revised genomic organization of $\mathrm{FBN} 1$ and significance for regulated gene expression. Genomics 1999;56:70-7.

7 Krawczac M, Cooper D. The human genome database. Trends Genet 1997;13:121-2.

8 Collod-Beroud G, Beroud C, Ades L, Black C, Boxer M, Brock DJ, Holman KJ, de Paepe A, Francke U, Grau U, Hayward C, Klein H, Liu W, Nuytinck L, Peltonen L, Perez ABA, Rantamaki T, Junien C, Boileau C. Marfan Database (third edition): new mutations and new routines for the software. Nucleic Acids Res 1998;26:229-3.

9 Loeys B, Nuytinct L, Delvaux I, De Bie S, De Paepe A. Genotype and phenotype analysis of 171 patients referred for molecular study of the fibrillin-1 gene FBN1 because of suspected Marfan syndrome. Arch Intern Med 2001; 12:2447-54

10 Korkko J, Kaitila I, Lonnquist L, Peltonen L, Ala-Kokko L. Sensitivity of conformation sensitive gel electrophoresis in detecting mutations in Marfan syndrome and related conditions. J Med Genet 2002;39:34-41.

11 Tynan K, Comeau K, Pearson M, Wilgenbus P, Levitt D, Gasner C, Berg MA, Miller DC, Francke U. Mutation screening of complete fibrillin-1 coding sequence: report of five new mutations, including two in 8-cysteine domains. Hum Mol Genet 1993:2:1813-21.

12 Kainulainen K, Karttunen L, Puhakka L, Sakai L, Peltonen L. Mutations in the fibrillin gene responsible for dominant ectopia lentis and neonatal Marfan syndrome. Nat Genet 1994;6:64-9.

13 Nijbroek G, Sood S, McIntosh I, Francomano CA, Bull E, Pereira L, Ramirez F, Pyeritz RE, Dietz HC. Fifteen novel FBN1 mutations causing Marfan syndrome detected by heteroduplex analysis of genomic amplicons. Am J Hum Genet 1995;57:8-21

14 Liu W, Qian C, Comea K, Bren T, Furthmayr H, Francke U. Mutant fibrillin-1 monomers lacking EGF-like domains disrupt microfibril assembly and cause severe Marfan syndrome. Hum Mol Genet 1996:5:1581-7.

15 Hayward C, Brock DJ. Fibrillin-1 mutations in Marfan syndrome and other type-1 fibrillinopathies. Hum Mutat 1997; 10:415-23.

16 Liu WO, Oefner PJ, Qian C, Odom RS, Francke U. Denaturing HPLC-identified novel FBN1 mutations, polymorphisms, and sequence variants in Marfan syndrome and related connective tissue disorders. Genet Test 1997;1:237-42. 
17 Comeglio P, Evans AL, Brice GW, Child AH. Detection of six novel FBN1 mutations in British patients affected by Marfan syndrome. Hum Mutat $2001 ; 18: 251$.

18 Booms P, Cisler J, Mathews KR, Godfrey M, Tiecke F, Kaufmann UC, Vetter U, Hagemeier C, Robinson PN. Novel exon skipping mutation in the fibrillin-1 gene: two 'hot spots' for the neonatal Marfan syndrome. Clin Genet 1999;55:1 10-17.

19 Schrijver I, Liu W, Brenn T, Furthmayr H, Francke U. Cysteine substitutions in epidermal growth factor-like domains of fibrillin-1: distinct effects on biochemical and clinical phenotypes. Am J Hum Genet 1999;65: 1007-20

20 Dietz HC, Mclntosh I., Sakai LY, Corson GM, Chalberg SC, Pyeritz RE, Francomano CA. Four novel FBN1 mutations: significance for mutant transcript level and EGF-like domain calcium binding in the pathogenesis of Marfan syndrome. Genomics 1993;17:468-75.

21 Dietz HC, Pyeritz RE, Puffenberger EG, Kendzior RJ, Corson GM, Maslen CL, Sakai LY, Francomano CA, Cutting GR. Marfan phenotype variability in a family segregating a missense mutation in the epidermal growth factor-like motif of the fibrillin gene. J Clin Invest 1992;89: 1674-80.

22 Montgomery RA, Geraghty MT, Bull E, Gelb BD, Johnson M, McIntosh I, Francomano CA, Dietz HC. Multiple molecular mechanisms underlying subdiagnostic variants of Marfan syndrome. Am J Hum Genet 1998:63:1703-11.

23 Halliday D, Hutchinson S, Kettle S, Firth H, Wordsworth P, Handford PA. Molecular analysis of eight mutations in FBN1. Hum Genet 1999; 105:587-97.

24 Hutchinson S, Wordsworth P, Handford P. Marfan syndrome caused by a mutation in FBN1 that gives rise to cryptic splicing and a 33 nucleotide insertion in the coding sequence. Hum Genet 2001;109:416-20.

25 Hayward C, Rae AL, Porteous ME, Logie LJ, Brock DJ. Two novel mutations and a neutral polymorphism in EGF-like domains of the fibrillin gene (FBN1): SSCP screening of exons 15-21 in Marfan syndrome patients. Hum Mol Genet 1994;3:373-5.

26 Yuan B, Thomas JP, von Kodolitsch Y, Pyeritz RE. Comparison of heteroduplex analysis, direct sequencing, and enzyme mismatch cleavage for detecting mutations in a large gene, FBN1. Hum Mutat 1999:14:440-6

27 Matyas G, De Paepe A, Halliday D, Boileau C, Pals G, Steinmann B. Evaluation and application of denaturing HPLC for mutation detection in Marfan syndrome: identification of 20 novel mutations and 2 novel polymorphisms in the FBNI gene. Hum Mutat 2002:19:443-56.

28 Kainulainen K, Karttunen L, Puhakka L, Sakai L, Peltonen L. Mutations in the fibrillin gene responsible for dominant ectopia lentis and neonatal Marfan syndrome. Nat Genet 1994;6:64-9
29 Lonnquist L, Child A, Kainulainen K, Davidson R, Puhakka L, Peltonen L. A novel mutation of the fibrillin gene causing ectopia lentis. Genomics 1994; 19:573-6.

30 Pepe G, Giusti B, Evangelisti L, Porciani MC, Brunelli T, Giurlani L, Attanasio M, Fattori R, Bagni C, Comeglio P, Abbate R, Gensini GF. Fibrillin-1 (FBN1) gene frameshift mutations in Marfan patients: genotype-phenotype correlation. Clin Genet 2001;59:444-50

31 Liu W, Smith DI, Rechtzigel KJ, Thibodeau SN, James CD. Denaturing high performance liquid chromatography (DHPLC) used in the detection of germline and somatic mutations. Nucleic Acids Res 1998;26:1396-400.

32 O'Donovan MC, Oefner PJ, Roberts SC, Austin J, Hoogendoorn B, Guy C, Speight G, Upadhyaya M, Sommer SS, McGuffin P. Blind analysis of denaturing high-performance liquid chromatography as a tool for mutation detection. Genomics 1998;52:44-9.

33 Toudiarska I, Kilpatrick MW, Lembessis P, Carra S, Harton GL, Sisson ME, Black SH, Stern HJ, Gelman-Kohan Z, Shohat M, Tsipouras P. Novel approach to the molecular diagnosis of Marfan syndrome: application to sporadic cases and in prenatal diagnosis. Am J Med Genet 2001;99:294-302.

34 Silverman DI, Burton KJ, Gray J, Bosner MS, Kouchoukos NT, Roman M, Boxer M, Devereux RB, Tsipouras P. Life expectancy in the Marfan syndrome. Am J Cardiol 1995;75:157-60.

35 Tiecke F, Katzke S, Booms P, Robinson PN, Neumann L, Godfrey M, Mathews KR, Scheuner M, Hinkel GK, Brenner RE, Hovels-Gurich HH, Hagemeier C, Fuchsc J, Skovby F, Rosenberg T. Classic, atypically severe and neonatal Marfan syndrome: twelve mutations and genotype-phenotype correlations in FBN1 exons 24-40. Eur J Hum Genet 2001;9:13-21

36 Godfrey $M$, Vandemark $N$, Wang $M$, Velinov $M$, Wargowski $D$, Tsipouras P, Han J, Becker J, Robertson W, Droste S, Rao VH. Prenatal diagnosis and a donor splice site mutation in fibrillin in a family with Marfan syndrome. Am J Hum Genet 1993;53:472-80.

37 Pereira L, Levran O, Ramirez F, Lynch JR, Sykes B, Pyeritz RE, Dietz HC. A molecular approach to the stratification of cardiovascular risk in families with Marfan's syndrome. N Engl J Med 1994;331:148-53.

38 Mottes M, Mirandola S, Rigatelli F, Zolezzi F, Lisi V, Gordon D, Pignatti PF. Allelic frequencies of FBN1 gene polymorphisms and genetic analysis of Italian families with Marfan syndrome. Hum Hered 2000;50:175-9.

39 Roman MJ, Devereux RB, Kramer-Fox R, Loughlin J. Two-dimensional echocardiographic aortic root dimensions in normal children and adults. Am J Cardiol 1989:64:507-12

40 Ahn NU, Sponseller PD, Ahn UM, Nallamshetty L, Rose PS, Buchowski JM, Garrett ES, Kuszyk BS, Fishman EK, Zinreich SJ. Dural ectasia in the Marfan syndrome: MR and CT findings and criteria. Genet Med 2000;2:173-9.

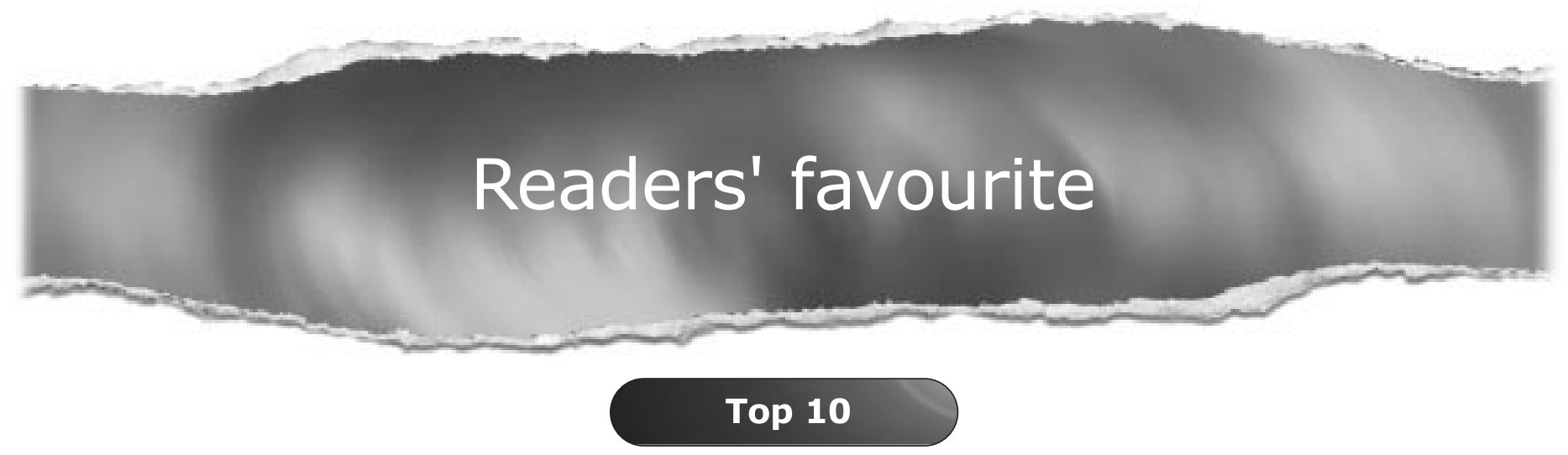

Click on the "Top 10" button on the homepage to see which are the best read articles each month

www.jmedgenet.com 